\title{
Green Synthesis and Characterization of Silver Nanoparticles using Cinnamomum zeylanicum Bark Extract
}

\author{
C. T. Supritha ${ }^{1 *}$, S. S. Chandrashekhar ${ }^{1}$, P. U. Krishnaraj ${ }^{2}$, \\ Ravi Hunje ${ }^{1}$ and N. S. Hebsur ${ }^{3}$
}

${ }^{1}$ Department of Seed Science and Technology, ${ }^{2}$ Department of Agricultural Microbiology, ${ }^{3}$ Department of Soil Science and Agricultural Chemisrty, AC, UAS, Dharwad, India

*Corresponding author

\section{A B S T R A C T}

\section{Keywords}

Cinnamon, Green

synthesis,

Nanoparticles,

Silver

Article Info

Accepted:

04 December 2020

Available Online:

10 January 2021
An experiment on green synthesis of silver nanoparticles and their characterization was carried out during 2018 to 2020 at University of Agricultural Sciences, Dharwad. Green synthesis of silver nanoparticles (AgNPs) was done using bark extract of Cinnamomum zeylanicum. Characterization was done through UVVisible spectrophotometer, Particle Size Analyzer, Atomic Force Microscope, Scanning Electron Microscope, Energy-dispersive X-ray spectroscopy and X-ray Diffraction. The biosynthesised AgNPs showed absorption peak at $428.7 \mathrm{~nm}$ with an average particle size of $38.5 \mathrm{~nm}$. SEM revealed that nanoparticles were spherical in shape and EDAX confirmed presence of silver ions in biosynthesized sample. AFM confirmed a smooth and monodispersed particles and XRD showed crystalline structure of nanoparticles.

\section{Introduction}

Technology related to measure, manipulation or incorporation of materials of size ranging from $1 \mathrm{~nm}$ to $100 \mathrm{~nm}$ is known as Nanotechnology. The nanoparticles are made up of thousands of atoms and can be considered to be larger than atoms and most of the molecules. Though it is a recent field of science, its application is increasing day by day. Its applications can be found in many areas such as industry, agriculture, business, medicine, public health due to its high demand. The use of plants as a production medium of silver nanoparticles has drawn attention because of its rapid, and presence of various metabolites that may aid in reducing silver and most importantly it is ecofriendly, non-pathogenic, economical protocol and providing a single step technique for the biosynthetic processes

In the present work, biosynthesis of silver nanoparticles (AgNPs) using the commercially economic and abundantly available Cinnamomum zeylanicum bark as the biomaterial. $C$. zeylanicum bark was selected because of its high antioxidant 
activity (Shan et al., 2007) and flavonoid content.In addition to synthesis, extensive characterization of the synthesized AgNPs using UV-Visible spectrophotometer and dynamic light scattering method were also studied to investigate the impact of synthesis conditions on the yield, physiochemical features, and stability of the nanoparticles. Scanning Electron Microscopy (SEM) and Xray diffraction (XRD) were used to analyze the morphology of the nanoparticles.

\section{Materials and Methods}

The experiments were conducted in the Green Nanotechnology Laboratory, Microbial Genetics Laboratory, Seed Quality Research Laboratory of National Seed Project and Department of Seed Science and Technology, University of Agricultural Sciences, Dharwad.

An experiment on green synthesis of silver nanoparticles using three different sources and study their effects on onion (Allium cepa L.) seed quality enhancement was carried out during April 2018 to September 2020.

\section{Preparation of bark powder aqueous extracts}

The C. zeylanicum bark rolls were collected from local grocery store in raw form and air dried in hot air oven, then grinded to fine powder which was used for preparation of aqueous solution. $10 \mathrm{~g}$ of the prepared powder was mixed in $200 \mathrm{~mL}$ of deionized distilled water and heated on a water bath at $60{ }^{\circ} \mathrm{C}$ for 15 minutes.

The extract solution was cooled at room temperature and filtered using Whatman filter paper No. 1 and the filtered mixture was stored in refrigerator at $4{ }^{\circ} \mathrm{C}$ for further utilization in the experiments.
Preparation of $1000 \mathrm{~mL}$ aqueous $\mathrm{AgNO}_{3}$ (300 ppm) stock solution

Silver nitrate $\left(\mathrm{AgNO}_{3}\right)$ procured from Sigma Aldrich (USA) was used as a precursor in the synthesis of silver nanoparticles. $0.29 \mathrm{~g}$ of $\mathrm{AgNO}_{3}$ was dissolved in $1000 \mathrm{~mL}$ of deionized water and stored in yellow colored bottle for use in further experiments.

Biosynthesis of silver nanoparticles by Cinnamomum zeylanicum

A total of $1 \mathrm{~mL}$ of the C. zeylanicum bark aqueous extract was added to $100 \mathrm{~mL}$ of silver nitrate solution (300 ppm). This mixture was exposed to bright sunlight for 40 minutes, the colour of the mixture changed from light brown to dark brown (Fig. 1) which indicated the formation of silver nanoparticles. The reduction of $\mathrm{Ag}^{+}$was analysed preliminarily by UV-visible (Vis) spectrophotometry. This synthesized AgNPs was stored at $-80{ }^{\circ} \mathrm{C}$ for further experiments involving nanopriming of onion seeds. For characterization of synthesized AgNPs, the solution containing the formed AgNPs was centrifuged at 15,000 rpm for 10 minutes followed by drying in vacuum oven at $35{ }^{\circ} \mathrm{C}$ for 48 hours and the pellet was collected and stored.

\section{Characterization of synthesized silver nanoparticles}

\section{UV-Visible spectroscopy}

Reduction of $\mathrm{Ag}^{+}$ions to AgNPs was confirmed by UV-Vis spectrophotometer (SP-UV 500DB, Germany) at scanning wavelengths of 200-700 $\mathrm{nm}$ based on their optical absorbance peak. As the size of the nanoparticles decreases, the band gap increases and thus, the optical absorbance increases as compared to that of the bulk particles and therefore their colour changes. The synthesized AgNPs was first sonicated 
using solid probe Ultra sonicator (inkarp 20 $\mathrm{kHz} \pm 50 \mathrm{~Hz}$. Sonics Vibracell) for uniform distribution of nanoparticles. Then diluted five times with deionized distilled water before spectrophotometric measurement and an aliquot of extract was used as control and the absorbance maxima was recorded at room temperature.

\section{Particle Size Analyzer (PSA)}

The NPs were further subjected to dynamic light scattering (DLS) to measure average particle size, in a high-performance particlesize analyzer (model Z3000, Nicomp, USA). The sample was diluted before placing in disposable polystyrene cuvettes and the analysis was carried out at room temperature in triplicate with a scattering angle of $90^{\circ}$.

\section{Atomic Force Microscope (AFM)}

Silver nanoparticles topography was examined by AFM (Nanosurf AG, Swiss, Flex AFM with 24 bit C3000). AFM is ideal for qualitatively measuring surface roughness and visualizing the surface texture of nanoparticles. It has a very high three dimensional spatial resolution. The surface of the sample is scanned using a probe and the oscillation amplitude is used to measure the surface characteristics of the sample.

The AgNPs samples were sonicated for 30 minutes and thin film was coated on mica sheet and allowed to dry for few minutes at room temperature and left overnight in hot air oven at $30^{\circ} \mathrm{C}$. Then again samples were kept under room condition for ten minutes before placing under AFM. Silver nanoparticles were characterized by AFM in dynamic mode by using NCLR-10 cantilever and the 2-D and 3$\mathrm{D}$ images of nanoparticles with respect to size and distribution was observed and further analysis was done by nanosurf software.

\section{Scanning Electron Microscope (SEM)}

The silver nanoparticles were then subjected to surface morphology analysis by scanning electron microscopy (SEM; Carl Zeiss, Germany). Nanoparticles were placed on an aluminum holder with carbon adhesive tape and SEM images were captured with an EVO 18 microscope operated with smart SEM software.

The presence of $\mathrm{Ag}^{+}$ions was further confirmed by energy-dispersive X-ray spectroscopy (EDS), attached to the EVO 18 scanning electron microscope. The pellets obtained by centrifugation of synthesized NPs were powdered and a pinch of sample was uniformly placed on an aluminum stub and coated with gold in a sputter coater and examined using SEM at different voltages at a working distance between $8-9 \mathrm{~mm}$ and a voltage of $5-10 \mathrm{kV}$.

\section{X-ray diffraction (XRD)}

The reduced AgNPs powder was subjected to X-ray diffraction (XRD) pattern and crystalline structure was examined by using the Rigaku TTR (Tokyo, Japan) at 40KV and $150 \mathrm{~mA}$ within $2 \mathrm{~h}$ area between 10 and 90 with an intensity $\mathrm{Cu} \mathrm{K} \alpha$ line $(\lambda=0.15406$ $\mathrm{nm})$.

\section{Results and Discussion}

\section{Characterization of synthesized nanoparticles}

Protocol for green synthesis of silver nanoparticles using bark extract of Cinnamomum zeylanicum

The protocol for green synthesis of AgNPs using bark extract of $C$. zeylanicum was standardized after repeated trials involving varied quantity of aliquots and exposure time. 
Based on the varied quantities of aliquot and different duration of sunlight exposure, the procedure for green synthesis of AgNPs using bark extract of $C$. zeylanicum was standardised.

In addition, the reaction mixture was evaluated at various time intervals of exposure to bright sunlight and agitation using ultrasonic probe. The maximum number of $\mathrm{Ag}^{+}$ions reduced to $\mathrm{Ag}^{0}$ in the $\mathrm{AgNO}_{3}$ solution (Fig. 2) at $40 \mathrm{~min}$ of sunlight exposure $\left(100 \mathrm{~mL} \mathrm{AgNO}_{3}\right.$ and $1 \mathrm{~mL}$ bark extract). The size of the synthesized AgNPs was confirmed by DLS which showed mean distribution of $39 \mathrm{~nm}$.
The preliminary confirmation of formation of nanoparticles was crudely done by observation of change in colour of reaction mixture by the end of the reaction. The AgNPs syntheised using bark extract of $C$. zeylanicum was light golden color at the beginning of reaction which ultimately turned to dark brown after $40 \mathrm{~min}$ of sunlight exposure indicating the end of reaction that is maximum reduction of $\mathrm{Ag}^{+}$ions to $\mathrm{Ag}^{0}$. The increase in the absorbance values with increased dosage up to $1 \mathrm{~mL}$ and incubation of reaction mixture for the biosynthesis process also demonstrated higher production of AgNPs, which is due to the availability of more reducing biomolecules for the reduction of silver ions at this dosage (Table 1).

Table.1 Protocol for synthesis of AgNPs using bark extract of C. zeylanicum

\begin{tabular}{|c|c|c|c|c|}
\hline $\begin{array}{c}\text { Aqueous AgNO } \\
\mathbf{3 0 0} \mathbf{~ p p m}(\mathbf{m L})\end{array}$ & $\begin{array}{c}\text { Bark extract } \\
(\mathbf{m L})\end{array}$ & $\begin{array}{c}\text { Sunlight exposure } \\
\text { (minutes) }\end{array}$ & $\begin{array}{c}\text { Ultra sonication } \\
\text { (minutes) }\end{array}$ & $\begin{array}{c}\text { Particle size } \\
\text { analyser }(\mathbf{n m})\end{array}$ \\
\hline $\mathbf{1 0 0}$ & 10 & 30 & 20 & 952 \\
\hline $\mathbf{1 0 0}$ & 5 & 30 & 20 & 890 \\
\hline $\mathbf{1 0 0}$ & 2.5 & 30 & 20 & 789 \\
\hline $\mathbf{1 0 0}$ & 2.5 & 60 & 20 & 515 \\
\hline $\mathbf{1 0 0}$ & 2.5 & 60 & 40 & 765 \\
\hline $\mathbf{1 0 0}$ & 1.0 & 60 & 20 & 375 \\
\hline $\mathbf{1 0 0}$ & 1.0 & 60 & 30 & 251 \\
\hline $\mathbf{1 0 0}$ & 1.0 & 45 & 20 & 105 \\
\hline $\mathbf{1 0 0}$ & 2.5 & 40 & 20 & 88 \\
\hline $\mathbf{1 0 0}$ & 1.0 & 40 & 20 & 58 \\
\hline $\mathbf{1 0 0}$ & 1.0 & 60 & 20 & 74 \\
\hline $\mathbf{1 0 0}$ & 1.0 & 40 & - & 39 \\
\hline
\end{tabular}

Fig.1 Color change of reaction mixture

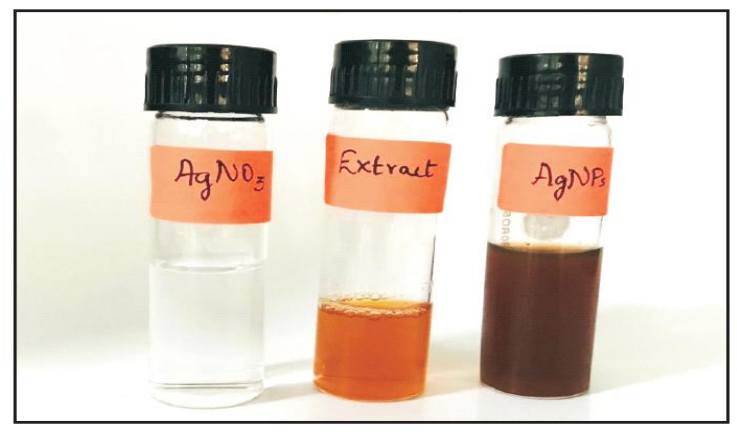


Fig.2 Flowchart of biosynthesis of AgNPs using bark extract Cinnamomum zeylanicum

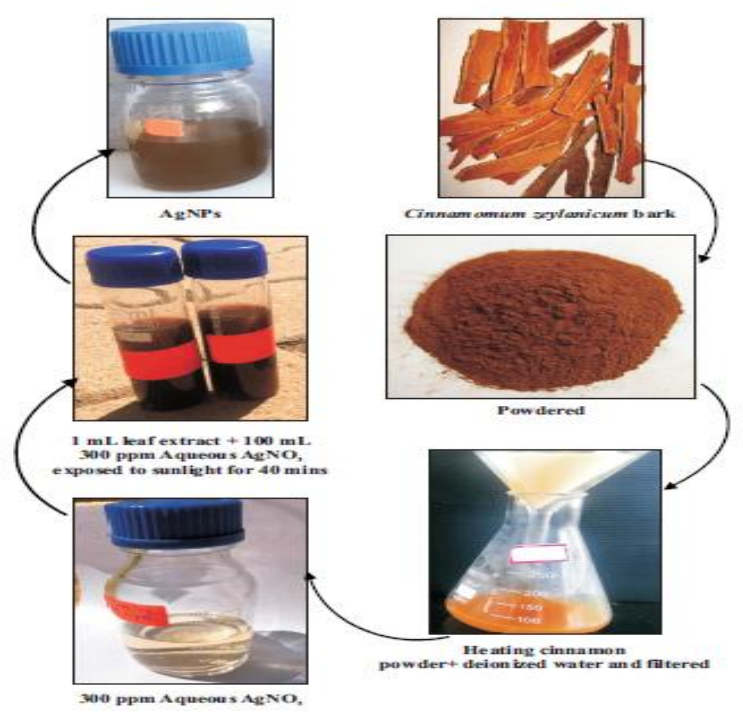

Fig.3 UV-Vis spectrophotometer reading of biosynthesised AgNPs

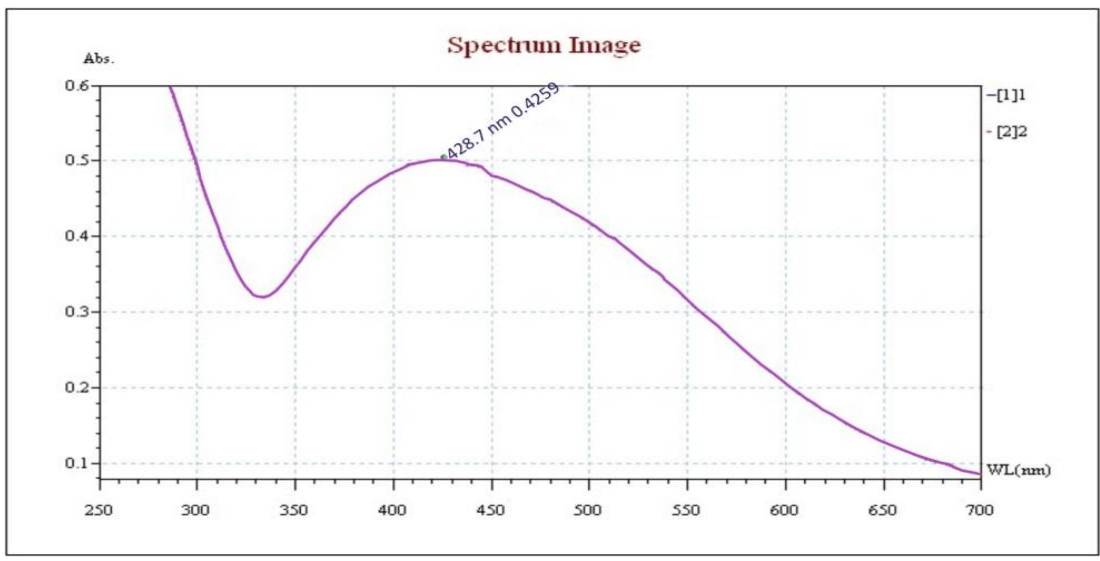

Fig.4 Particle size analyzer reading of biosynthesised AgNPs

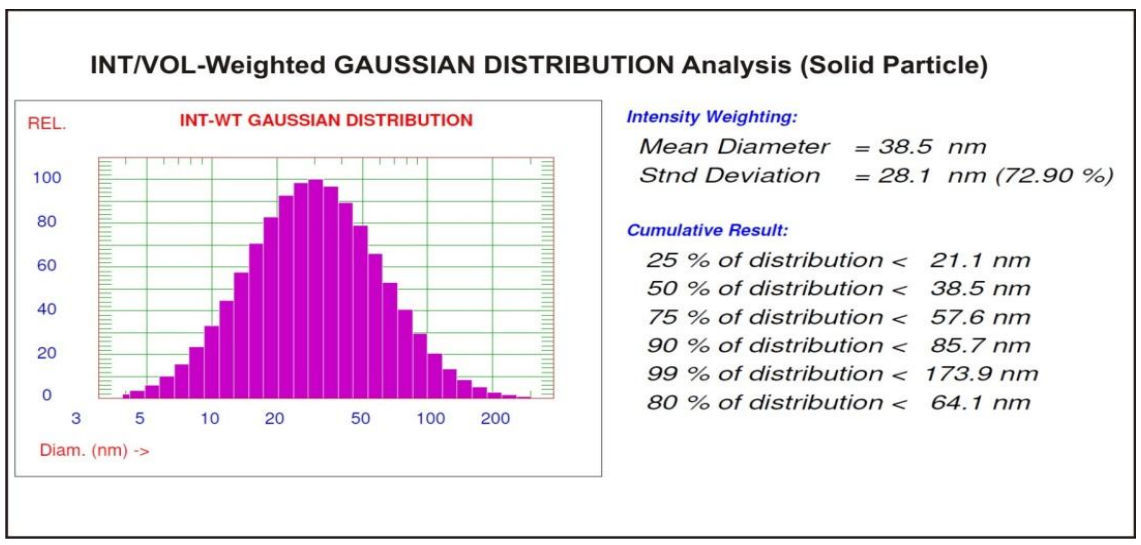


Fig.5 Two and three dimensional graphical images of biosynthesised AgNPs
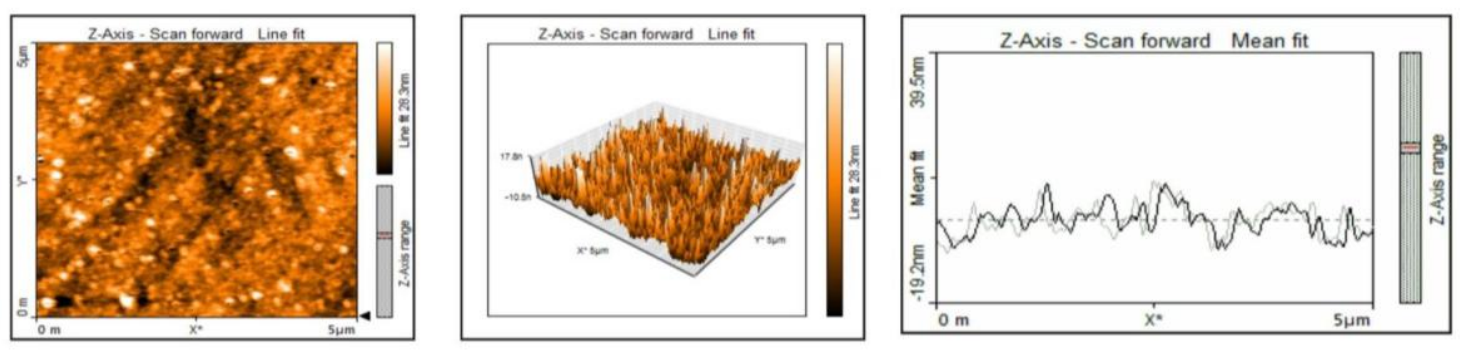

Fig.6 SEM images of biosynthesised AgNPs

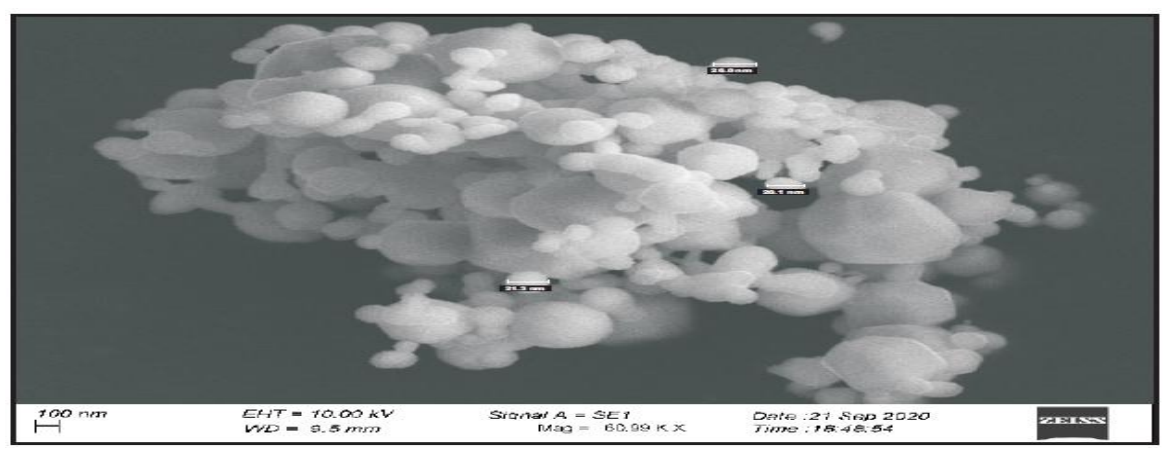

Fig.7 EDAX analysis graph of biosynthesised AgNPs

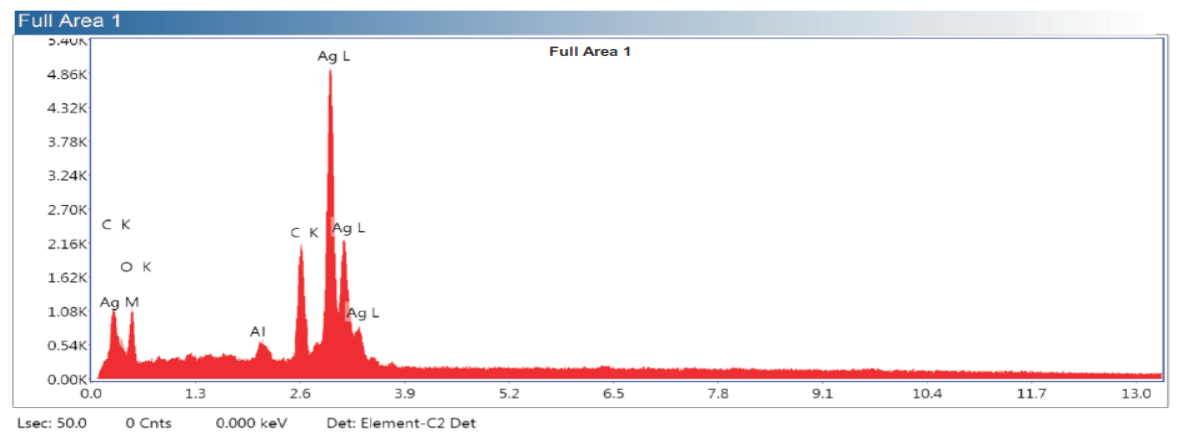

Fig.8 XRD graph images of biosynthesised AgNPs

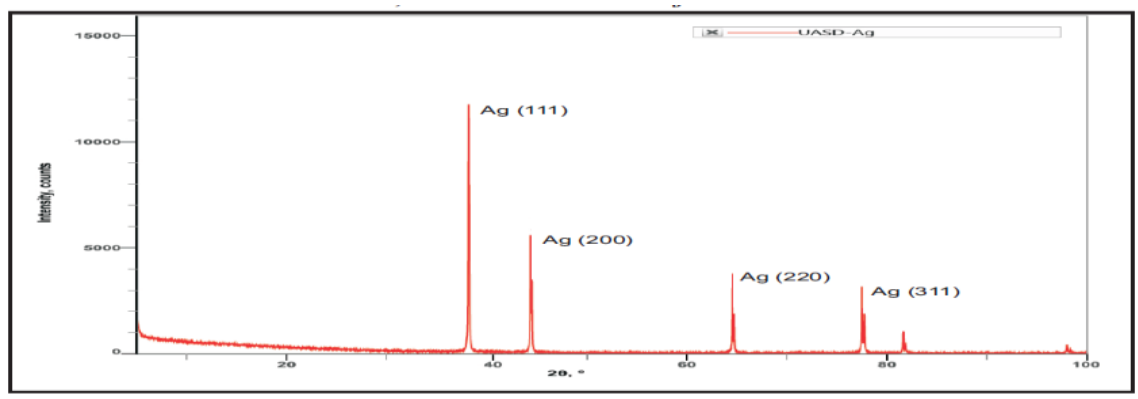


Similar results were reported by Huang et al., 2007. Terpenoids are believed to play an important role in biosynthesis of AgNPs through the reduction of Ag ions. Shankar et al., 2004 has reported the possibility of terpenoids from geranium leaf in the synthesis of nano-sized silver particles. AgNPs formation by bark extract as a reducing agent was confirmed by the absorption peak at $428.7 \mathrm{~nm}$ (Fig. 3) in the UV-Vis spectrophotometer. In this study, a strong and broad surface plasmon resonance peak 428 $\mathrm{nm}$ was obtained confirming the successful synthesis of AgNPs. Previous studies suggested that a surface plasmon resonance peak located between 410 and $450 \mathrm{~nm}$ exists for AgNPs and could be attributed to spherical shaped nanoparticles (Jyotiet al., 2016). The intensity of the color change was directly related to the incubation period and the extract used. This is probably due to the stimulation of $\mathrm{AgNO}_{3}$ reduction and plasmon vibrations (Manikandan et al., 2017 \& Masum et al., 2019). The DLS analysis yielded a mean diameter of $38.5 \mathrm{~nm}$ with 25 per cent of the distribution under $21.1 \mathrm{~nm}$ for AgNPs synthesized by bark extract of $C$. zeylanicum (Fig. 4). The per cent distribution in relation to intensity-weight reflects the average size of AgNPs and a range between lowest and highest size with level of uniformity in synthesized AgNPs. These results are in line with the study conducted by Sandeep et al., (2017). The AgNPs synthesized were characterized by AFM in relation with shape, size, 2D and 3D images of the nanoparticles in dynamic mode by using NCLR- 10 cantilever. The surface topography or the morphology, dispersion and aggregation of nanoparticles were revealed by AFM using the 3D images produced showed a formation of homogeneous distribution with no agglomeration. The synthesized nanoparticles had a medium rough surface with spherical shaped nanoparticles (Fig. 5). The combination of SEM with energy- dispersive X-ray spectroscopy (EDX) was used to examine silver powder morphology and also conduct chemical composition analysis information regarding the purity and the degree of particle aggregation. The nanoparticles of various sizes were located with the smooth shiny surfaces and spherical in shape (Fig. 6). Similarly the elemental composition of all three AgNPs were confirmed by X-ray analysis using the EDS system attached to the SEM. EDS spectral images showed peak at $2.98 \mathrm{keV}(\mathrm{L} \alpha)$, confirming the presence of elemental Ag. The EDAX signals were captured at the higher peaks marked the presence of $\mathrm{Ag}$ in the AgNPS synthesized (Fig. 7). The diffraction profile had an intense peak at $2 \theta$ of $38.85^{\circ}$, $43.04^{\circ}, 64.25^{\circ}$ and $83.34^{\circ}$ corresponding to (111), (200), (220) and (311) planes in AgNPs reduced by $C$. zeylanicum bark extract (Fig. 8).

In conclusion the green nanotechnology is a flourishing field generating interest among researchers towards eco-friendly biosynthesis of nanoparticles and having a huge potential to bring benefits in areas of interfacing physical, chemical, biological, agricultural and environmental sciences with myriad applications. The monodispersed silver nanoparticles synthesised using $C$. zeylanicum are stable and eco-friendly.

\section{References}

Huang, J., Li, Q., Sun, D. and Lu, Y., (2007) Biosynthesis of silver and gold nanoparticles by novel sundries Cinnamomum camphora leaf. Nanotechnology 18 (10): 104-105.

Jyoti, K., Baunthiyal, M. and Singh, A., (2016). Characterization of silver nanoparticles synthesized using Urticadioica L. leaves and their synergistic effects with antibiotics. Journal of Radiation 
Research and Applied Sciences. 9(3): 217-227.

Manikandan, R., Beulaja, M., Thiagarajan, R., Palanisamy, S., Goutham, G., Koodalingam, A., Prabhu, N. M., Kannapiran, E., Basu, M. J., Arulvasu, C. and Arumugam, M., (2017) Biosynthesis of silver nanoparticles using aqueous extract of Phyllanthus acidus L. fruits and characterization of its anti-inflammatory effect against $\mathrm{H} 2 \mathrm{O} 2$ exposed rat peritoneal macrophages. Process Biochemistry 55: 172-181.

Masum, M., Islam, M., Siddiqa, M., Ali, K. A., Zhang, Y., Abdallah, Y., Ibrahim, E., Qiu, W., Yan, C. and Li, B., (2019) Biogenic synthesis of silver nanoparticles using Phyllanthus emblica fruit extract and its inhibitory action against the pathogen Acidovorax oryzae Strain RS-2 of rice bacterial brown stripe. Frontier Microbiolology. 10: 820.

Sandeep, D., (2017). Green synthesis of nanoparticles and their effect on seed quality and longevity of soybean (Glycine max).Ph.D. Thesis, Uni. Agri. Sci., Dharwad (India).

Shan, B., Cai, Y. Z., Brooks, J. D. and Corke, H., (2007) Antibacterial properties and major bioactive components of cinnamon stick (Cinnamomum burmannii): activity against foodborne pathogenic bacteria. Journal of Agriultural. Food and chemistry. 55(14): 5484-5490.

Shankar, S. S., Rai, A., Ankamwar, B., Singh, A., Ahmad, A. and Sastry, M., (2004) Biological synthesis of triangular gold nanoprisms. Nature Materials. 7: 482-488.

\section{How to cite this article:}

Supritha, C. T., S. S. Chandrashekhar, P. U. Krishnaraj, Ravi Hunje and Hebsur, N. S. 2021. Green Synthesis and Characterization of Silver Nanoparticles using Cinnamomum zeylanicum Bark Extract. Int.J.Curr.Microbiol.App.Sci. 10(01): 444-451. doi: https://doi.org/10.20546/ijcmas.2021.1001.055 